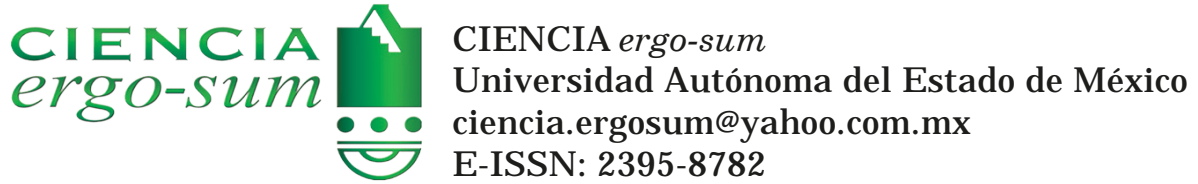

\title{
Mejoramiento de la calidad en la carne de la trucha arcoíris mediante la técnica de sacrificio I kejime: caso Ecuador
}

\section{Salazar-Duque, Diego; Holguín, J uan Pablo; Estrella I., Alexisy Lomas Martínez, Geovanny}

Mejoramiento de la calidad en la carne de la trucha arcoíris mediante la técnica de sacrificio I kejime: caso Ecuador CIE NCIA ergo-sum, vol. 26, núm. 1, marzo-junio 2019|e45

Universidad Autónoma del Estado de México, México

Esta obra está bajo una Licencia Creative Commons Atribución-NoComercial-SinDerivar 4.0 Internacional .

Salazar-Duque, D., Holguín, J. P., Estrella I., A. y Lomas Martínez, G. (2019). Mejoramiento de la calidad en la carne de la trucha arcoíris mediante la técnica de sacrificio I kejime: caso Ecuador. CI E N CI A ergo-sum, 26(1). https://doi.org/10.30878/ces.v26n1a10 


\title{
Mejoramiento de la calidad en la carne de la trucha arcoíris mediante la técnica de sacrificio Ikejime: caso Ecuador
}

Improvement of rainbow trout's meat quality through slaughter Ikejime technique. Study case: Ecuador

\author{
Diego Salazar-Duque \\ Universidad UTE, Ecuador \\ diegoa.salazar@ute.edu.ec \\ Juan Pablo Holguin \\ Universidad UTE, Ecuador \\ juanp.holguin@ute.edu.ec \\ Alexis Estrella I. \\ Universidad UTE, Ecuador \\ alexis.estrella.i@gmail.com \\ Geovanny Lomas Martinez \\ Investigador independiente \\ geo.lomas@hotmail.com
}

Recepción: 6 de abril de 2018

Aprobación: 29 de junio de 2018

\section{Resumen}

Las técnicas de sacrificio empleadas tradicionalmente en la industria pesquera provocan niveles altos de estrés en los peces debido a la violencia del proceso. Esta condición influye en la calidad de su carne. Con estos antecedentes, se identifica la técnica de sacrificio más adecuada para preparaciones culinarias mediante una evaluación sensorial para los atributos de olor, sabor y textura que permita potenciar esa calidad. Según las referencias recopiladas de carácter empírico, la técnica Ikejime, de origen japonés, es la más idónea para sacrificar peces. Las características gastronómicas de la carne de los que fueron pescados por este medio son superiores respecto a los que no. Las pruebas realizadas comprobaron dichas aseveraciones.

Palabras Claves: trucha arcoíris, técnicas de sacrificio: Ikejime, congelación, análisis sensorial.

\begin{abstract}
The slaughter techniques traditionally used in the fishing industry cause high levels of stress in fish due to the violence of the process. This condition directly influences the fish meat quality. The objective of this study was to identify the most suitable slaughter technique for culinary preparations by a sensorial evaluation focused on odor, flavor and texture attributes. According to the empirical references collected, the Ikejime technique of Japanese origin is the most appropriate to slaughter fish. The gastronomic characteristics of fish which were killed by this technique, are superior to fish that suffered another type of death. The carried out tests verified these assertions.
\end{abstract}

KEYWORDS: rainbow trout, slaughter techniques: Ikejime, freezing, sensory analysis.

\section{INTRODUCCIÓN}

Una de las principales causas que puede influir en la calidad de la carne de un pescado, utilizada en el arte culinario, es el nivel de estrés que se produce durante su sacrificio. Según Diaz-Villanueva y Robotham (2015), el estrés en los peces se encuentra asociado a la producción de radicales libres que promueven la oxidación de las grasas, lo cual conlleva a obtener una calidad baja en su carne. De acuerdo con De la Gándara (2003), un pez 
que muere luchando bajo condiciones de estrés presenta niveles más altos de ácido láctico en su carne, los cuales motivan un descenso del $\mathrm{pH}$, condición ideal para la proliferación de bacterias que aceleraran el proceso de putrefacción. Con esto, el principal objetivo del artículo es identificar la técnica más adecuada y recomendable para sacrificar la trucha arcoíris que permita aprovechar de forma efectiva su carne como producto alimenticio en la preparación de platillos gastronómicos.

Existen algunas referencias técnicas y científicas para sacrificar a los pescados y la técnica japonesa Ikejime es una de las más aconsejables. Lamentablemente, la información bibliográfica es muy escasa, ya que la mayor parte de las fuentes se basa en información obtenida de la práctica empírica que realizan pescadores japoneses. Una de las referencias que se pueden citar sobre las bondades de esta técnica es la del chef argentino Andrés Medici del restaurante Osushi (Saber y Sabor, 2014). Otra referencia es de los autores Myhrvold y Young (2010), quienes manifiestan que la aplicación de la técnica es un mecanismo para provocar el menor dolor y estrés posible en el pez durante su sacrificio para obtener una carne de calidad. Este estudio se sustenta en los resultados presentados por Auró y Ocampo (1999) sobre el estrés que pueden sufrir los peces durante su muerte; por lo tanto, se desarrolla un estudio empírico a través de la técnica de sacrificio Ikejime. Para poder comprobar estos estudios, tanto desde el punto de vista técnico como gastronómico, se presentan las siguientes hipótesis:

a) H1 La técnica de sacrificio Ikejime supera de forma positiva el tiempo de muerte en la trucha arcoíris a diferencia de la técnica producida por asfixia.

b) H2 La técnica de sacrificio Ikejime supera de forma positiva el tiempo de muerte en la trucha arcoíris a diferencia de la técnica producida por congelación.

c) H3 La técnica de sacrificio Ikejime en la trucha arcoíris es el procedimiento más adecuado para las preparaciones culinarias.

\section{Trucha ARcoíris}

Ancorbynchus mykiss, conocida comúnmente como trucha arcoíris por los puntos multicolores de su piel, pertenece a la familia Salmonidae originaria del Océano Pacífico, desde Japón hasta México (Jiménez Prado et al., 2015). Una empresa canadiense la introdujo a Ecuador en 1928 y seleccionó diversos ríos, riachuelos y lagos a lo largo de la región interandina para potencializar la pesca deportiva y la acuicultura (Mora y Uyaguari, 2004). Entre sus cualidades está la de adaptarse a zonas geográficas que alcanzan altitudes superiores a los $3000 \mathrm{msnm}$ (Barriga, 2012). El atractivo de esta especie radica en la coloración de su carne, que va desde tonos rosas a rojos, la cual se da por la absorción y depósito de pigmentos carotenoides oxigenados en sus músculos (Pokniak et al., 2001).

Estudios realizados por Coll (1999) identifican a la trucha arcoíris como un pez teleósteo procedente de aguas frías y carnívoro; su fuente de alimentación está basada en peces nativos más pequeños, anfibios y ciertos invertebrados como son los camarones de río (Barriga, 2012). En la actualidad, existe una población muy numerosa que requiere de un gran volumen de comida; esto genera la extinción de algunas especies endémicas de algunos ríos ubicados en los Andes ecuatorianos, como es el caso de la preñadilla (Balarezo, 2014), razón por la cual está considerada dentro de las 100 especies exóticas más dañinas e invasoras del mundo (Lowe et al., 2000).

Para cubrir la creciente demanda de trucha arcoíris que se requiere para consumo humano en el mercado nacional ecuatoriano, la Subsecretaría de Acuacultura de Ecuador ha dotado de alevines genéticamente mejorados para el repoblamiento de ríos, lagos y lagunas (Ministerio de Agricultura, G. A., 2015a; 2015b). A la fecha, en el país existen 213 criaderos de truchas distribuidos en las provincias de Azuay, Bolívar, Cañar, Carchi, Chimborazo, Cotopaxi, Imbabura, Loja, Napo, Pichincha, Sucumbíos y Tungurahua, con una producción de 982.3 toneladas anuales (Buenaño, 2010). 


\section{TÉCNICAS DE SACRIFICIO}

Se consideran técnicas de sacrificio a los procedimientos por los cuales un individuo busca dar muerte a un animal con el fin de obtener provecho. Según la Autoridad de Seguridad Alimentaria Europea (EFSA, por sus siglas en inglés), se utilizan diversas técnicas de aturdimiento y sacrificio en truchas arcoíris, las cuales pueden ser practicadas en forma artesanal y profesional. Entre los ejemplos más comunes se encuentran métodos de percusión, métodos eléctricos, dióxido de carbono, desangramiento, congelación y asfixia (EFSA, 2009), además de las generadas por otras culturas a partir de sus creencias o posibilidades técnicas. Estas tres últimas técnicas, por poseer una fuerte representatividad en el arte culinario, serán consideradas en este estudio.

\section{1. Técnica de sacrificio por asfixia}

Los peces, al igual que los seres humanos, necesitan de oxígeno para vivir. El oxígeno disuelto en el agua es captado por los peces: al abrir y cerrar la boca hacen circular el agua que atraviesa las cámaras branquiales captando el oxígeno que es dirigido a la sangre (Ommanney, 1964). Este complejo sistema torna imposible la supervivencia de los peces al exponerse al aire. Por lo tanto, este tipo de muerte por asfixia conlleva dolor. De acuerdo con Huntingford (2006), los peces tienen la capacidad de sufrir y detectar estímulos de dolor, en donde sus vías sensoriales procesan dichos estímulos, codifican dicha información y generan respuestas comportamentales muy agitadas.

\section{2. Técnica de sacrificio por congelación}

Los peces que se sumergen en agua a temperaturas inferiores a $0^{\circ}$ Celsius mueren porque se torna imposible su adaptación. Según estudios realizados por Fletcher et al. (1988), la temperatura letal para la trucha arcoíris es de $-0.75^{\circ} \mathrm{C}$; dicha temperatura provoca un shock térmico (hipotermia) en el animal y su tiempo de muerte dependerá de factores como peso, edad y sexo. Si bien la hipotermia es reconocida como un método de anestesia que tranquiliza al pez, las bajas temperaturas aumentan la capacidad para transportar el oxígeno, por lo que se reduce la actividad móvil, la respiración, la sensibilidad y la estimulación (De la Gándara, 2003) e impide alcanzar un nivel real de analgésico y se genera cierto estrés en el animal.

\section{3. Técnica de sacrificio Ikejime}

Este método japonés sirve para matar un pez mediante el corte y la destrucción de su cordón espinal; aunque podría resultar un método cruel, es todo lo contrario: su procedimiento es rápido y genera el mínimo dolor en el animal (Myhrvold y Young, 2010). De acuerdo con Van De Vis et al. (2003), este proceso se efectúa en cuatro pasos: $a$ ) aturdimiento, $b$ ) desangre, $c$ ) desprendimiento de la médula espinal y $d$ ) anulación del estrés durante el sacrificio.

El primer paso (aturdimiento) tiene como objetivo principal provocar una pérdida de conciencia y de sensibilidad (Diaz-Villanueva y Robotham, 2015). Al introducir una pica de hielo en el cerebro, se anula la respuesta del hipotálamo en el pez, ya que pierde los estímulos nerviosos como dolor, miedo, ansiedad, hambre, frío, calor. Según Ommanney (1964), un pez posee todos los sentidos, además de un "sexto sentido", el cual se genera por medio de órganos nerviosos situados bajo la piel al percibir estímulos externos producidos por cambios bruscos en el entorno, y que se transforman en señales que son dirigidas al hipotálamo, encargado de segregar hormonas de estrés como respuesta.

El segundo paso (desangre) consiste en que, aún vivo el pez y antes que aparezca el rigor mortis (FAO, 1998), se debe expulsar la sangre del animal (Gil Cano et al., s.f.). Este procedimiento dentro del arte culinario permite 
obtener filetes blancos y uniformes. Para realizar el desangre, hay que hacer dos cortes que permitan la expulsión de la sangre de manera violenta. El primer corte se realiza con la ayuda de un cuchillo a la altura de las branquias; esta incisión busca lesionar las arterias aorta dorsal y ventral. El segundo corte se localiza en la cola del pez, donde la arteria caudal es la afectada en ese paso (figura 1).

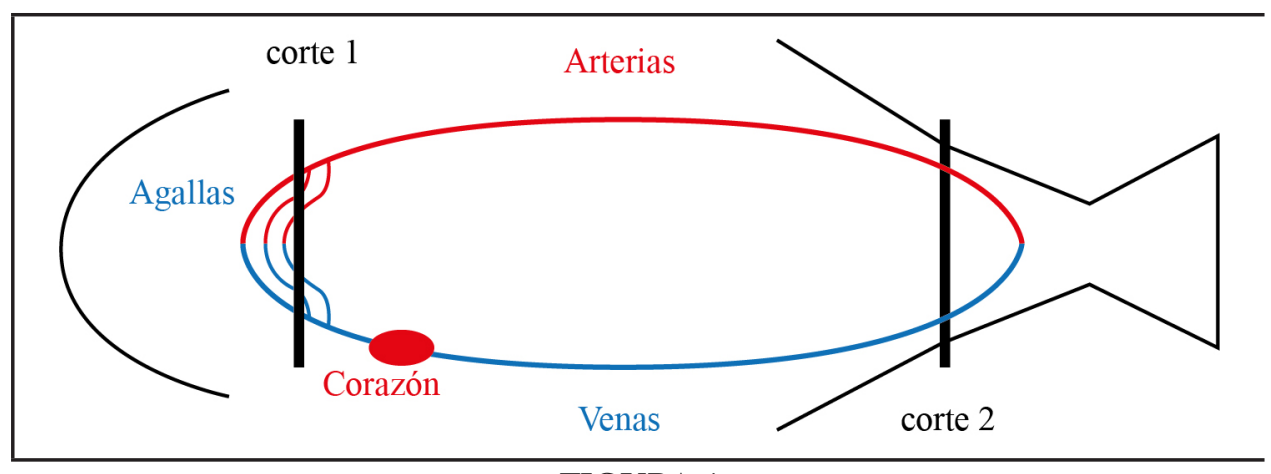

FIGURA 1

Sistema circulatorio del pez

Fuente: elaboración propia.

En el tercer paso (desprendimiento) se debe retirar la médula espinal que se encuentra dentro de la columna vertebral del pez, la cual está compuesta por una serie de discos conformados por huesos y cartílagos. La médula se encarga de llevar los pulsos nerviosos generados por el hipotálamo a todo el cuerpo. Dentro del Ikejime, este paso consiste en introducir un cable sólido a través de la médula con el fin de desprenderla y eliminar el canal por el cual se transmiten los pulsos nerviosos.

Finalmente, en el cuarto paso (anulación del estrés durante el sacrificio), se reduce el grado de estrés en el pez al estar en un estado de inconsciencia previo a la muerte, por lo que no puede sentir ninguna sensación de excitación, dolor o pánico.

De acuerdo con Auró y Ocampo (1999), se pueden encontrar varias circunstancias que generan estrés en el animal y que tendrán un efecto negativo en la calidad de la carne. Por ejemplo:

a) Estrés social: provocado por las altas densidades de la población en las piscinas.

b) Estrés físico: causado por cambios en la temperatura del agua, niveles de oxígeno, $\mathrm{pH}$ del agua, traumatismos provocados por golpes entre la población, exposición al aire, descompresión atmosférica, captura, hacinamiento y transporte.

c) Estrés nutricional: causado por la deficiencia o exceso de algún nutriente en la dieta.

Entre las variables que emplearon Auró y Ocampo (1999) para diagnosticar el tipo de estrés, se encuentran la medición de variables bioquímicas en la sangre y tejidos, modificaciones en las respuestas fisiológicas y la imagen de células y tejidos, liberación de adrenalina, noradrenalina, cortisol, alteración glicemia, ácido láctico, glucógeno hepático y muscular, alteración en el número de células rojas y blancas, alteración en la concentración plasmática del cloro, sodio y potasio y cambios en el comportamiento.

Algunos resultados encontrados por Auró y Ocampo (1999) en la trucha arcoíris señalan que a) la exposición a temperaturas entre los 30 y $45^{\circ} \mathrm{C}$ produce cambios autolíticos en el páncreas (estrés físico), $b$ ) un efecto brusco del $\mathrm{pH}$ del agua provoca cambios negativos en la piel (estrés físico) y $c$ ) una alimentación con ácidos grasos no saturados o deficientes en vitamina E conlleva una panseatitis o inflamación de la grasa corporal (estrés nutricional). Hay algunas ventajas del Ikejime que se pueden apreciar haciendo un símil con la carne de res. Un toro de lidia presenta una carne de baja calidad debido a aspectos como el estrés, las lesiones y el intenso trabajo muscular que resulta en el aumento del pH por falta de ácido láctico y en la retención de agua, lo cual predispone 
a la proliferación bacteriana, además de presentar carnes duras, secas y oscuras. Todo esto en respuesta a una lucha hasta la extenuación dentro de un ambiente extraño (Pozo, 1993).

En un pez puede suceder lo mismo: empiezan a actuar factores como falta de oxígeno, la presión atmosférica y los golpes que pueda recibir en la superficie donde es colocado al ser extraído del agua; si se emplea anzuelo, el dolor que éste provoca, más los golpes contra otros peces, genera un alto nivel de estrés. El esfuerzo del organismo soporta secreción de adrenalina y noradrenalina (hormonas de estrés). La cantidad de catecolaminas en el torrente circulatorio presenta un resultado negativo en la calidad de la carne (Pozo, 1993). El efecto de la noradrenalina y adrenalina provoca un aumento del ritmo cardiaco que a su vez aumenta la presión sanguínea y, en consecuencia, lesiones y pequeñas hemorragias (Pozo, 1993). La contracción muscular del pez al estar expuesto al aire determina un gasto de la energía que tiene como reserva (glucógeno). Al reducirse la cantidad de glucógeno, existe una menor generación de ácido láctico mediante la glucogenolisis anaeróbica. El ácido láctico estimula que la carne baje su pH y la torna ácida. Si el pez consume sus reservas energéticas, habrá menor cantidad de ácido láctico y la carne tendrá un $\mathrm{pH}$ alto provocando una alteración microbiana. Esta técnica busca precisamente eso: reducir el estrés durante la muerte y con ello las implicaciones anotadas. El Ikejime busca sacrificar al pez empleando la menor cantidad de tiempo posible, así como evitar su sufrimiento.

\section{Evaluación Sensorial}

La evaluación sensorial, según el Instituto de Alimentos de EE.UU. es la disciplina científica utilizada para medir, analizar e interpretar las reacciones o características de los alimentos que son percibidas por los sentidos: vista, olfato, gusto, tacto y oído (Institute of Food, 2014). Para la evaluación sensorial, se requieren varias técnicas de tal manera que las respuestas de los evaluadores sean lo más precisas posible (Ramírez- Navas, 2012).

La manera de medir la sensación es por medio de métodos psicológicos (Sancho, 2002). Las sensaciones pueden percibirse a través de uno o varios sentidos y al miso tiempo son codificadas por el cerebro de acuerdo con la información que posea de un alimento. El cerebro asocia sabores, texturas, pero sobre todo aromas o recuerdos que se tienen de un alimento; se podría afirmar que las cualidades de los alimentos son una invención del cerebro (Sáez, 2016). Todo este proceso que se da dentro de la mente tiene como resultado una aceptación o rechazo de un alimento, un efecto producido por la lógica sensorial del cerebro gastronómico que posee cada individuo.

Para Ramírez-Navas (2012), las pruebas de análisis sensorial tienen como objetivo la definición clara de los atributos que tiene un alimento. Para esto, se aplica una metodología conocida como pruebas orientadas al consumidor. En estas pruebas, el evaluador da su opinión del producto y de esta manera define sus características (Ramírez-Navas, 2012).

\section{Metodología}

El desarrollo de esta investigación es de naturaleza descriptivo-experimental con un diseño multivariado. Se enfoca en elementos que tienen una estrecha relación con las técnicas de sacrificio en animales para consumo humano, en especial aquellas técnicas que son generadas por asfixia, congelación e Ikejime (técnica japonesa). La metodología empleada para este estudio fue establecida en cinco fases: a) selección de la especie, $b$ ) sacrificio, $c$ ) cocción, $d$ ) prueba de aceptabilidad y e) evaluación de resultados. Para la selección de la especie, se establecieron muestras de Ancorhynchus mykiss que fueron recolectadas en las piscinas del criadero "El Pedregal” y están ubicadas en la provincia de Cotopaxi a una altura de 3800 metros. Los prototipos fueron distribuidos en tres rangos de peso: de 200 a 300 gramos, de 301 a 400 gramos y de 401 a 500 gramos. Para evaluar el sacrificio, se utilizó, en primera instancia, la técnica de asfixia en tres tipos de ejemplares, uno de cada rango de peso establecido. Dicho proceso consistió en exponer al animal a una muerte natural, que fue 
sacarlo de la piscina de criadero al ambiente. Para la técnica de congelación, se expusieron las tres muestras a una temperatura de $-7.7^{\circ} \mathrm{C}$ hasta su muerte. En cuanto a la técnica Ikejime, las tres muestras fueron tratadas con un cuidado especial para evitar el estrés en el animal y se aplicó el procedimiento con celeridad. Para evaluar los tiempos de muerte, según las diferentes técnicas, se utilizó una escala de muerte entre 1 y 25 minutos, en rangos de 5 minutos cada uno, con una escala de valoración de 5 puntos. Esto permitió identificar la relación entre el tiempo de muerte y la técnica de sacrificio empleada. El instrumento empleado fue un cronómetro. Los resultados se analizaron mediante el cálculo de la moda estadística con una hoja de cálculo de Excel. El cuadro 1 muestra la escala de valoración.

\section{CUADRO 1}

Escala de valoración del tiempo de muerte

\begin{tabular}{|lll}
\hline Puntaje & Valoración & \multicolumn{1}{c}{ Tiempo } \\
\hline 5 & excelente & entre 1 y 5 minutos \\
4 & muy bueno & entre 6 y 10 minutos \\
3 & bueno & entre 11 y 15 minutos \\
2 & regular & entre 16 y 20 minutos \\
1 & malo & más de 21 minutos \\
\hline
\end{tabular}

Fuente: elaboración propia.

Una vez aplicada cada técnica de sacrificio, las truchas fueron lavadas y conservadas a $0{ }^{\circ} \mathrm{C}(\mathrm{FAO}, 1995)$ por un periodo de 24 horas para luego ser sometidas a cocción. Durante este procedimiento, se sometió a un proceso de limpieza en el que se retiró cabeza, cola, espinas y piel y posteriormente se sumergieron en una salmuera (disolución de sal en agua) al 10\% de concentración (del peso total del pescado se utiliza la misma cantidad de agua, la suma de estos dos representa $100 \%$ y con este valor se determina la cantidad de sal) con el fin de realzar el sabor y mejorar las propiedades naturales de la carne durante la cocción (este efecto mantiene la cantidad de agua propia del pescado dentro de sí mismo y aporta jugosidad).

Una vez realizado lo anterior, se sometió a un proceso de cocción con el objeto de que sean bromatológicamente aptas para el consumo (Ferre, 2001) mediante una técnica de vacío que consiste en colocar los filetes de trucha dentro de bolsas plásticas, extraer el oxígeno, sellarlas de manera hermética con una máquina VacMaster VP215 al $99 \%$ y, para terminar, someterlas a baño María a una temperatura controlada de $55^{\circ} \mathrm{C}$. El cuadro 2 muestra los tiempos de cocción empelados durante el estudio.

\section{CUADRO 2}

Tiempos de cocción de los objetos de estudio

\begin{tabular}{|lccc}
\hline Objeto de estudio & $\begin{array}{c}\text { Peso } \\
\text { gramos }\end{array}$ & $\begin{array}{c}\text { Temperatura de cocción } \\
\text { Celsius }\end{array}$ & $\begin{array}{c}\text { Tiempo } \\
\text { minutos }\end{array}$ \\
\hline OE 1.1 & 131 & $55^{\circ} \mathrm{C}$ & 5 \\
OE 1.2 & 91 & $55^{\circ} \mathrm{C}$ & 5 \\
OE 1.3 & 120 & $55^{\circ} \mathrm{C}$ & 5 \\
OE 2.1 & 135 & $55^{\circ} \mathrm{C}$ & 8 \\
OE 2.2 & 130 & $55^{\circ} \mathrm{C}$ & 8 \\
OE 2.3 & 167 & $55^{\circ} \mathrm{C}$ & 8 \\
OE 3.1 & 224 & $55^{\circ} \mathrm{C}$ & 10 \\
OE 3.2 & 220 & $55^{\circ} \mathrm{C}$ & 10 \\
OE 3.3 & 190 & $55^{\circ} \mathrm{C}$ & 10 \\
\hline
\end{tabular}

Fuente: elaboración propia

Nota: OE (objeto de estudio); 1, n (tanque 1); 2, n (tanque 2); 3, n (tanque 3). 
Para la evaluación sensorial, se utilizó una prueba de aceptabilidad conocida como nivel de agrado (Ramírez-Navas, 2012) que consiste en determinar si existen diferencias entre las muestras que se evalúan a través de escalas hedónicas de 3, 5, 7 y 9 puntos. Para el ejercicio, se empleó una escala hedónica de 5 puntos, un modelo aplicado en estudios por Quitral (2015), donde se evalúan atributos como olor, sabor y textura (Ramírez-Navas, 2012). La tabla 2 muestra el grado de evaluación que se empleó durante el estudio con una escala de 5 puntos. Para establecer la relación existente entre los atributos (olor, sabor, textura) respecto de las técnicas de sacrificio empleadas en las truchas, se realizó un análisis de varianza (ANOVA) acompañado de la prueba Post Hoc con los test de Tukey y Games-Howell para identificar si existen diferencias significativas; para ello, se empleó el programa estadístico SPSS 23 para Windows.

TABLA 2

Escala hedónica para la evaluación sensorial para los atributos olor, sabor y textura

\begin{tabular}{|c|c|}
\hline Puntaje & Nivel de agrado \\
\hline 5 & me gusta mucho \\
\hline 4 & me gusta \\
\hline 3 & no me gusta ni me disgusta \\
\hline 2 & me disgusta \\
\hline 1 & me disgusta mucho \\
\hline
\end{tabular}

Fuente: elaboración propia.

Tomando en cuenta que muchas veces el producto escogido por el evaluador experto no es necesariamente el elegido por el consumidor, se consideran los siguientes aspectos en cuanto a la determinación del panel de evaluadores y así reducir la subjetividad de la prueba:

a) El perfil que debían presentar los catadores debía ser restringido, donde los gustos, la expectación y la objetividad se considerarían como los factores psicológicos más importantes (Lund et al., 2009).

b) Seleccionar los evaluadores de acuerdo con su agudeza sensorial básica y su capacidad para describir sus percepciones en forma razonada, que no se deje influir por prejuicios (FAO, 2009).

c) Escoger un líder de panel entrenado que pueda ser objetivo y capaz de utilizar diferenciaciones amplias en cuanto al uso de terminología (Muñoz, 1998).

d) Determinar para el resto de panelistas un equipo de gastrónomos expertos, vinculados al área de la educación, con capacidad de diferenciar factores de calidad en rangos mínimos, de percibir y describir de forma coherente los gustos que acompañan los defectos en pescado y los productos pesqueros; capaz de definir los estímulos sensoriales y vincularlos a una causa subyacente en el producto (FAO, 2009).

Entre los elementos que coadyuvaron a la objetividad de la prueba, se presentaron las muestras en platos de porcelana, cubiertos y un vaso con agua. Además, se escogió un espacio que estuviera aislado de ruidos, sin ventanas y con luz tenue para minimizar los distractores. El fin de este proceso era evaluar las distintas muestras presentadas al azar indicando el grado de aceptabilidad.

\section{Resultados}

\section{1. Tiempo de muerte}

Entre los primeros resultados presentados se encuentra el que hace referencia a la relación entre el tipo de muerte, empleando diversas técnicas de sacrificio (asfixia, congelación e Ikejime), y el tiempo que demoró 
en morir el objeto de estudio (trucha). Como se puede observar en el cuadro 3, los objetos de estudio en los que se empleó la técnica asfixia $(1.1,2.1,3.1)$ alcanzaron una valoración, a partir de la moda, de 3 puntos equivalente a bueno. En el grupo de peces con los que se utilizó la congelación (1.2, 2.2, 3.2), la valoración de la moda fue de 4 puntos, equivalente a muy buena; finalmente, la técnica de sacrificio Ikejime (1.3, 2.3. 3.3) obtuvo una valoración de la moda de 5 puntos y cada ejemplar recibió una valoración de excelente.

Los resultados muestran una diferencia gradual en la valoración de tiempo de muerte en los peces. Al analizar los datos, se observó que los peces sacrificados con la técnica Ikejime mueren en un lapso de 5 minutos; en contraposición con la técnica de asfixia que recibió un puntaje de 3 , donde el tiempo de muerte fue entre 10 a 15 minutos, lo cual indica que es la que más tiempo emplea para que el pez muera. Este resultado permite corroborar la hipótesis $\mathrm{H} 1$ y $\mathrm{H} 2$ de forma afirmativa respectivamente.

CUADRO 3

Valoración de los tiempos de muerte

\begin{tabular}{lcccccccccc}
\hline Tipo de sacrificio & 1.1 & 1.2 & 1.3 & 2.1 & 2.2 & 2.3 & 3.1 & 3.2 & 3.3 & Moda \\
\hline Asfixia & 3 & & & 3 & & & 3 & & & 3 \\
Congelación & & 4 & & & 4 & & & 4 & & 4 \\
Ikejime & & & 5 & & & 5 & & & 5 & 5 \\
\hline
\end{tabular}

Fuente: elaboración propia.

\section{2. Evaluación sensorial}

Los resultados del análisis sensorial para el atributo olor se muestran en el cuadro 4 y cuadro 5 . Para establecer si existen diferencias significativas, el valor de la significancia (Sig.) debe ser menor a 0.05. Los resultados de la prueba ANOVA para este estudio sobre el atributo olor, respecto a cada una de las técnicas de sacrificio empleadas, da una significancia (Sig.) de 0.771. Esto determina que no existen diferencias significativas para el atributo olor.

\section{CUADRO 4}

Análisis ANOVA para el atributo olor empleando como técnicas de sacrificio la asfixia, la congelación e Ikejime

\begin{tabular}{lcrccc}
\hline & Sum of Squares & $d f$ & Mean Square & $F$ & Sig. \\
\hline Between Groups & 0.454 & 2 & 0.227 & 0.262 & 0.771 \\
Within Groups & 34.662 & 40 & 0.867 & & \\
Total & 35.116 & 42 & & & \\
\hline
\end{tabular}

Fuente: elaboración propia.

Nota: para establecer si existen diferencias significativas, el valor de la significancia (Sig.) debe ser menor a 0.05 .

Para identificar las diferencias que se obtuvieron en el estudio para el atributo olor, comparando las tres técnicas de sacrificio, se procedió a la prueba Post Hoc (cuadro 5). Si la significancia (Sig.) es menor a 0.05 determina diferencias significativas. El cuadro 5 demuestra que comparando los resultados entre asfixia-congelación, asfixia-Ikejime, congelación-Ikejime, para el atributo olor, no existen diferencias significativas, ya que los valores de significancia son mayores a 0.05. Los resultados del análisis sensorial para el atributo sabor se muestran en el cuadro 6 y el cuadro 7.

Los resultados de la prueba ANOVA, para el atributo sabor respecto a cada una de las técnicas de sacrificio empleadas, arrojan una significancia (Sig.) de 0.442., lo cual determina que no existen diferencias significativas para el atributo sabor. 


\section{CUADRO 5}

Post Hoc para el atributo olor empleando como técnicas de sacrificio la asfixia, la congelación e Ikejime

\begin{tabular}{|c|c|c|c|c|c|c|c|}
\hline \multirow{2}{*}{$\begin{array}{l}\text { Dependent } \\
\text { variable: olor }\end{array}$} & \multirow{2}{*}{$\begin{array}{l}\text { (I) Técnica de } \\
\text { sacrificio }\end{array}$} & \multirow{2}{*}{$\begin{array}{l}\text { (J) Técnica de } \\
\text { sacrificio }\end{array}$} & \multirow{2}{*}{$\begin{array}{c}\text { Mean } \\
\text { Difference } \\
(I-J)\end{array}$} & \multirow[t]{2}{*}{ Std. Error } & \multirow[t]{2}{*}{ Sig. } & \multicolumn{2}{|c|}{ 95\% Confidence Interval } \\
\hline & & & & & & Lower Bound & Upper Bound \\
\hline \multirow{6}{*}{ Tukey HSD } & \multirow{2}{*}{ Asfixia } & Congelación & 0.010 & 0.346 & 1.000 & -0.83 & 0.85 \\
\hline & & Ikejime & 0.224 & 0.346 & 0.795 & -0.62 & 1.07 \\
\hline & \multirow{2}{*}{ Congelación } & Asfixia & -0.010 & 0.346 & 1.000 & -0.85 & 0.83 \\
\hline & & Ikejime & 0.214 & 0.352 & 0.816 & -0.64 & 1,07 \\
\hline & \multirow{2}{*}{ Ikejime } & Asfixia & -0.224 & 0.346 & 0.795 & -1.07 & 0.62 \\
\hline & & Congelación & -0.214 & 0.352 & 0.816 & -1.07 & 0.64 \\
\hline \multirow{6}{*}{ Games-Howell } & \multirow{2}{*}{ Asfixia } & Congelación & 0.010 & 0.358 & 1.000 & -0.88 & 0.90 \\
\hline & & Ikejime & 0.224 & 0.354 & 0.804 & -0.66 & 1.10 \\
\hline & \multirow[b]{2}{*}{ Congelación } & Asfixa & -0.010 & 0.358 & 1.000 & -0.90 & 0.88 \\
\hline & & Ikejime & 0.214 & 0.322 & 0.786 & -0.59 & 1.02 \\
\hline & \multirow{2}{*}{ Ikejime } & Asfixa & -0.224 & 0.354 & 0.804 & -1.10 & 0.66 \\
\hline & & Congelación & -0.214 & 0.322 & 0.786 & -1.02 & 0.59 \\
\hline
\end{tabular}

Fuente: elaboración propia.

Nota: para establecer si existen diferencias significativas, el valor de la significancia (Sig.) debe ser menor a 0.05.

\section{CUADRO 6}

ANOVA para el atributo sabor empleando como técnicas de sacrificio la asfixia, la congelación e Ikejime

\begin{tabular}{|lcrccc}
\hline & Sum of Squares & $d f$ & Mean Square & $F$ & Sig. \\
\hline Between Groups & 1.436 & 2 & 0.718 & 0.833 & 0.442 \\
Within Groups & 34.471 & 40 & 0.862 & & \\
\hline Total & 35.907 & 42 & & & \\
\hline
\end{tabular}

Fuente: elaboración propia.

Nota: para establecer si es que existen diferencias significativas el valor de la significancia (Sig.) debe ser menor a 0.05 .

\section{CUADRO 7}

Post Hoc para el atributo sabor empleando como técnicas de sacrificio la asfixia, la congelación e Ikejime

\begin{tabular}{|c|c|c|c|c|c|c|c|}
\hline \multirow{2}{*}{$\begin{array}{l}\text { Dependent } \\
\text { Variable: } \\
\text { sabor }\end{array}$} & \multirow{2}{*}{$\begin{array}{l}\text { (I) Técnica } \\
\text { de sacrificio }\end{array}$} & \multirow{2}{*}{$\begin{array}{l}\text { (J) Técnica } \\
\text { de sacrificio }\end{array}$} & \multirow{2}{*}{$\begin{array}{c}\text { Mean } \\
\text { Difference } \\
(I-J)\end{array}$} & \multirow[t]{2}{*}{ Std. Error } & \multirow[t]{2}{*}{ Sig. } & \multicolumn{2}{|c|}{$95 \%$ Confidence Interval } \\
\hline & & & & & & Lower Bound & Upper Bouna \\
\hline \multirow{6}{*}{ Tukey HSD } & \multirow{2}{*}{ Asfixia } & Congelación & -0.414 & 0.345 & 0.460 & -1.25 & 0.43 \\
\hline & & Ikejime & -0.057 & 0.345 & 0.985 & -0.90 & 0.78 \\
\hline & \multirow{2}{*}{ Congelación } & Asfixa & 0.414 & 0.345 & 0.460 & -0.43 & 1.25 \\
\hline & & Ikejime & 0.357 & 0.351 & 0.570 & -0.50 & 1.21 \\
\hline & \multirow{2}{*}{ Ikejime } & Asfixa & 0.057 & 0.345 & 0.985 & -0.78 & 0.90 \\
\hline & & Congelación & -0.357 & 0.351 & 0.570 & -1.21 & 0.50 \\
\hline \multirow{6}{*}{ Games-Howell } & \multirow{2}{*}{ Asfixia } & Congelación & -0.414 & 0.338 & 0.450 & -1.25 & 0.43 \\
\hline & & Ikejime & -0.057 & 0.365 & 0.987 & -0.96 & 0.85 \\
\hline & \multirow{2}{*}{ Congelación } & Asfixa & 0.414 & 0.338 & 0.450 & -0.43 & 1.25 \\
\hline & & Ikejime & 0.357 & 0.332 & 0.538 & -0.47 & 1.18 \\
\hline & \multirow{2}{*}{ Ikejime } & Asfixa & 0.057 & 0.365 & 0.987 & -0.85 & 0.96 \\
\hline & & Congelación & -0.357 & 0.332 & 0.538 & -1.18 & 0.47 \\
\hline
\end{tabular}

Nota: para establecer si es que existen diferencias significativas el valor de la significancia (Sig.) debe ser menor a 0.05 .

Fuente: elaboración propia. 
Para identificar las diferencias que se obtuvieron en el estudio para el atributo sabor, comparando las tres técnicas de sacrificio, se procedió a la prueba Post Hoc (cuadro 7). Si la significancia (Sig.) es menor a 0.05, determina diferencias significativas. El cuadro 7 arroja que comparando los resultados entre asfixia-congelación, asfixia-Ikejime, congelación-Ikejime para el atributo sabor no existen diferencias significativas, ya que los valores de significancia son mayores a 0.05 . Los resultados del análisis sensorial para el atributo textura se muestran en el cuadro 7 y el cuadro 8.

\section{CUADRO 8}

ANOVA para el atributo textura empleando como técnicas de sacrificio la asfixia, la congelación e Ikejime

\begin{tabular}{|lrrccc}
\hline & Sum of Squares & $d f$ & Mean Square & $F$ & Sig. \\
\hline Between Groups & 0.986 & 2 & 0.493 & 0.764 & 0.472 \\
Within Groups & 25.805 & 40 & 0.645 & & \\
\hline Total & 26.791 & 42 & & & \\
\hline
\end{tabular}

Nota: para establecer si es que existen diferencias significativas el valor de la significancia (Sig.) debe ser menor a 0.05 . Fuente: elaboración propia.

Los resultados de la prueba ANOVA para el atributo textura respecto a cada una de las técnicas de sacrificio empleadas da una significancia (Sig.) de 0.472 , lo cual determina que no existen diferencias significativas para el atributo sabor.

Para identificar las diferencias que se obtuvieron en el estudio para el atributo sabor, comparando las tres técnicas de sacrificio, se procedió a la prueba Post Hoc (cuadro 9). Si la significancia (Sig.) es menor a 0.05, determina diferencias significativas. El cuadro señala que comparando los resultados entre asfixia-congelación, asfixia-Ikejime, congelación-Ikejime para el atributo sabor no existen diferencias significativas, ya que los valores de significancia son mayores a 0.05 .

\section{CUADRO 9}

Post Hoc para el atributo textura empleando como técnicas de sacrificio la asfixia, la congelación e Ikejime

\begin{tabular}{|c|c|c|c|c|c|c|c|}
\hline \multirow{2}{*}{$\begin{array}{l}\text { Dependent } \\
\text { Variable: } \\
\text { textura }\end{array}$} & \multirow{2}{*}{$\begin{array}{l}\text { (I) Técnica } \\
\text { de sacrificio }\end{array}$} & \multirow{2}{*}{$\begin{array}{c}(J) \text { Técnica } \\
\text { de sacrificio }\end{array}$} & \multirow{2}{*}{$\begin{array}{c}\text { Mean } \\
\text { Difference } \\
(I-J)\end{array}$} & \multirow[t]{2}{*}{ Std. Error } & \multirow[t]{2}{*}{ Sig. } & \multicolumn{2}{|c|}{ 95\% Confidence Interval } \\
\hline & & & & & & Lower Bound & Upper Bound \\
\hline \multirow{6}{*}{ Tukey HSD } & \multirow{2}{*}{ Asfixia } & Congelación & -0.348 & 0.298 & 0.481 & -1.07 & 0.38 \\
\hline & & Ikejime & -0.276 & 0.298 & 0.628 & -1.00 & 0.45 \\
\hline & \multirow{2}{*}{ Congelación } & Asfixa & 0.348 & 0.298 & 0.481 & -0.38 & 1.07 \\
\hline & & Ikejime & 0.071 & 0.304 & 0.970 & -0.67 & 0.81 \\
\hline & \multirow{2}{*}{ Ikejime } & Asfixa & 0.276 & 0.298 & 0.628 & -0.45 & 1.00 \\
\hline & & Congelación & -0.071 & 0.304 & 0.970 & -0.81 & 0.67 \\
\hline \multirow{6}{*}{ Games-Howell } & \multirow{2}{*}{ Asfixia } & Congelación & -0.348 & 0.319 & 0.529 & -1.14 & 0.44 \\
\hline & & Ikejime & -0.276 & 0.295 & 0.624 & -1.01 & 0.46 \\
\hline & \multirow{2}{*}{ Congelación } & Asfixa & 0.348 & 0.319 & 0.529 & -0.44 & 1.14 \\
\hline & & Ikejime & 0.071 & 0.278 & 0.964 & -0.62 & 0.76 \\
\hline & \multirow{2}{*}{ Ikejime } & Asfixa & 0.276 & 0.295 & 0.624 & -0.46 & 1.01 \\
\hline & & Congelación & -0.071 & 0.278 & 0.964 & -0.76 & 0.62 \\
\hline
\end{tabular}

Nota: para establecer si es que existen diferencias significativas el valor de la significancia (Sig.) debe ser menor a 0.05 . Fuente: elaboración propia.

No se encuentran diferencias significativas en la calidad de la carne de pescado comparando las tres técnicas. La técnica de congelación obtiene resultados positivos (sin ser significativos); por lo tanto, se rechaza la hipótesis $\mathrm{H} 3$, que señalaba que la técnica de sacrificio Ikejime es el procedimiento más adecuado para las preparaciones culinarias. 


\section{Discusión}

Este artículo buscó comprobar las afirmaciones que se dan respecto a que el sacrificio de un pez empleando la técnica Ikejime provoca una muerte más rápida en comparación con otras técnicas. Al comparar el tiempo que emplea la técnica Ikejime con la congelación y asfixia (técnicas seleccionadas para el estudio), se logró comprobar la hipótesis respecto a la técnica con mejor tiempo para sacrificar peces, además se pudo establecer un orden dependiendo del tiempo que un pez tarda en morir después de aplicada la técnica. El orden es Ikejime, congelación y asfixia, lo cual se corrobora con los estudios de Auró y Ocampo (1999) y Myhrvold y Young (2010).

La segunda hipótesis que el estudio buscó determinar fue la influencia de la técnica de sacrificio en la calidad de la carne de pescado para preparaciones culinarias a través de un análisis sensorial que evaluaba los atributos de olor, sabor y textura. Los atributos evaluados no presentaron una diferencia estadística significativa según el análisis de varianza (ANOVA) y de comparación múltiple (Post Hoc) con un nivel de significancia de $p<0.05$. Sin embargo, los promedios generales reportan una preferencia en textura y sabor hacia los peces sacrificados por la técnica de congelación. Por lo tanto, esta investigación no comprobó las aseveraciones respecto a la técnica Ikejime comparando tal como lo manifiesta Medici Andrés (2014). En esa medida, no se puede generalizar una técnica de sacrificio para todos los animales, así como lo planteó Pozo (1993) al referirse al empleo del Ikejime en el toro.

Este estudio también abre nuevas líneas de investigación que permiten responder preguntas como la siguiente: ¿la técnica Ikejime brinda efectivamente mejores características físico- químicas a los pescados? Es posible que se requiera de una prueba bioquímica que mida los niveles de hormonas de estrés y que permita tener resultados precisos para el empleo de la técnica de sacrificio Ikejime. Esto ayudaría mucho a la calidad del producto procesado y sin procesar.

\section{AnÁLISIS PROSPECTIVO}

Como análisis prospectivo para este estudio se considera un enfoque analítico sobre la reflexión colectiva que se tendría a futuro para optimizar el proceso de sacrificio de la trucha. Para esto, se toma como aclaración la importancia que genera la venta de pesca a nivel nacional e internacional.

Por un lado, al ser el Ecuador un país pesquero y consumidor de productos procedentes del mar, existe una gran cantidad de empresas que compran y venden pescado para consumo humano a nivel local, lo cual generaría de esta forma una demanda alta de productos con calidad. Por otro lado, se encuentra también el proceso de la pesca como uno de los principales rubros de exportación que tiene el Ecuador, puesto que vende y distribuye sus productos internacionalmente, donde los destinos principales son la Unión Europea y EE.UU., seguido de los mercados asiáticos (Ministerio de Comercio Exterior, 2017). Con este antecedente, se considera que un producto que pasa por un proceso de calidad debe poseer ciertos estándares que permitan ofertar un producto en óptimas condiciones para consumo inmediato, y más aún si pasa por un cambio de temperatura durante su traslado. La preservación de texturas y sabores en los pescados debe ser un valor extra que proporcione el país hacia el mercado local o internacional. Como bien se sabe, por ejemplo, el pescado ha sido parte de la tradición culinaria del Japón, donde el proceso de pesca, mediante la meticulosa práctica Ikejime, brinda ese aporte cultural y económico hacia la sociedad. La demanda del mercado japonés por pescados sacrificados con esta técnica lleva a un incremento en el precio de los ejemplares de hasta un 50\%. Prospectivamente, existe la posibilidad de exportar pescados al mercado internacional aplicando la técnica Ikejime y así favorecer a la calidad del producto y la economía del país. Con estos antecedentes, se mencionan a continuación los ámbitos que se deben reforzar para alcanzar este objetivo. 
a) Ámbito económico

Expandir la venta en el mercado nacional e internacional promocionando la calidad de pescado que se obtiene en el Ecuador mediante la creación de programas y proyectos que mejoren la prestación de servicios y los procesos de sacrificio de forma estandarizada, a partir de lo cual se fortalezcan los espacios para beneficio del sector público y privado a nivel nacional, zonal y local.

b) Ámbito formativo

Diseño e implementación de programas de faenamiento acuícola (pesquero) donde se aplique la técnica Ikejime que permita la venta y distribución de productos con calidad; de este modo, concientizar el producto que la sociedad va a comprar, vender y consumir.

c) Ámbito empresarial

Formar a los pesqueros, empresarios y distribuidores (Mipymes) que se dedican a este proceso de faenamiento para que entiendan la importancia que genera para el sector y para el país trabajar con un producto de calidad mediante la creación y mejora de las condiciones laborales de la industria pesquera ecuatoriana con el fin de crear nuevos procesos de pesca, comercialización y distribución de animales procedentes del mar o de los ríos de una forma consciente, sostenible y sustentable.

\section{Conclusiones}

Las técnicas de sacrificio de peces empleadas dentro de la industria pesquera van desde la asfixia (empleada principalmente en la pesca artesanal) a la congelación, en especial para peces de exportación. Son técnicas que conllevan tiempo para su desenlace, pero en ellas sobre todo los peces sufren niveles de estrés. La falta de oxígeno, sea por asfixia o congelación, dura aproximadamente 20 minutos hasta que el pez muere, tiempo en el cual se desarrollan reacciones neurológicas como la segregación de adrenalina, noradrenalina y cortisol (hormonas de estrés) que tienen una afectación directa en la calidad de la carne de pescado. Ikejime es una técnica japonesa que permite disminuir el tiempo que tarda el pez en morir; en menos de 5 minutos se lleva a cabo. Esta técnica reduce el estrés; por ende, la calidad de la carne de pescado tiene propiedades organolépticas mejores en comparación con los que son sacrificados con otras técnicas.

Las características que se obtuvieron con la cocción al vacío a $55^{\circ} \mathrm{C}$ fue una carne de pescado muy tierna, jugosa, con los miotomas fácilmente desprendibles. La aplicación de salmuera permite una salazón exacta, además de que ayuda a mantener la humedad dentro de las fibras tornando una suculencia agradable al paladar.

Los resultados obtenidos responden a una "lógica sensorial del cerebro". El cerebro asocia sabores, texturas, pero sobre todo aromas o recuerdos que se tiene de un alimento. Se podría afirmar que las cualidades de los alimentos son una invención del cerebro. Un pez que ha sido sacrificado mediante asfixia o congelación en su estructura muscular contiene partículas de sangre, fruto de un desangre incompleto. Con la técnica Ikejime las partículas de sangre disminuyen y el desangre es mayor en comparación con las técnicas de asfixia y congelación.

\section{ReFERENCIAS}

Auró, A., y Ocampo, L. (1999). Diagnóstico del estrés en peces. Veterinaria México, 30(4),337-344.

Balarezo, V. (23 de Abril de 2014). Disponible en http://www.pichincha.gob.ec/gestion/desarrollo-economico/unidades-desconcentradas/item/387-proyecto-prometeo-prenadilla.html. Consultado el 19 de febrero de 2016.

Barriga, R. (2012). Lista de peces de aguas dulces e itermareales del Ecuador. Revista Politécnica, 30(3), 83-119. 
Buenaño, M. (2010). Hemograma de trucha arcoiris (Oncorhynchus mikuss) en tres etapas de producción en la cuenca alta de la provincia del Napo, Ecuador. Boletín Técnico, 9, 1-14.

Coll, J. (1999). Acuicultura marina animal. Madrid: Ediciones Mundi-Prensa.

De la Gándara, F. (2003). ¿Son la tareas propias de un cultivo intensivo de peces mediterráneos compatibles con su bienestar? I Congreso Internacional sobre Bienestar Animal Murcia, 1-7.

Diaz-Villanueva, J. y Robotham, H. (2015). Comparación de dos métodos de sacrificio en trucha arcoiris. Latin American Journal of Aquatic Research, 43(2), 287-294.

EFSA (European Food Safety Authority). (2009). Species-specific welfare aspects of the main systems of stunning and killing of farmed fish: rainbow trout. EFS A Journal, 13.

FAO (Food and Agriculture Organization). (1995). Consultado el 12 de octubre de 2016. Disponible en http://www.fao.org/docrep/008/y5013s/y5013s03.htm

FAO (Food and Agriculture Organization). (1998). Consultado el 18 de febrero de 2016. Disponible en http:// www.fao.org/docrep/v7180s/v7180s04.htm

FAO (Food and Agriculture Organization). (2009). Proyecto de directricespara la evaluación sensorial del pescado y los mariscos en laboratorios. Consultado el 12 de octubre de 2016. Disponible en http://www.fao.org/ tempref/codex/Reports/Alinorm99/al99_18s.pdf

Ferre, I. (2001). Anisakosis y otras zoonosis parasitarias transmitidas por consumo. Revista Aquatic España, 14, 1-21.

Fletcher, G., Kao, M., y Dempson, B. (1988). Lethal freezing temperatures of Arctic char and other salmonids in the presence of ice. Aquaculture, 71(4),369-378.

Gil Cano, F., Ayala Florenciano, M. D. y López Albors, O. (s. f.). Corazón de los peces. Murcia: Universidad de Murcia.

Huntingford, F. A. (2006). Current issues in fish welfare. Journal of Fish Biology, 68(2), 332-372.

IFT (Technologists Institute of Food). (30 de enero de 2014). Consultado el 21 de diciembre de 2016. Disponible en www.ift.org

Jiménez Prado, P. J., Aguirre, W. E., Laaz Moncayo, E. D. y Navarrete, R. (2015). Guia de peces para aguas continentales en la vertiente occidental del Ecuador. Pontificia Universidad Católica del Ecuador Sede Esmeraldas.

Lowe, S., Browne, M., y Boudjelas, S. (2000). 100 of the world's worst invasive alien species a selection from the global invasive species. The invasive species specialist group.

Lund, C., Jones, V. y Spanitz, S. (2009). Effects and influences of motivation on trained panellists. Food Quality and Preference, 20, 295-303.

Medici Andrés (2014). Andrés Médici (Osushi): Investigo para hacer lo mismo que ayer pero mejor. Consultado el 9 de febrero de 2016. Disponible en https://cadenaser.com/ser/2014/02/24/gastro/1393204994_850215. html

Ministerio de Agricultura, G. A. (27 de octubre de 2015a). www.agricultura.gob.ec. Recuperado el 4 de febrero de 2016. Disponible en http://www.agricultura.gob.ec/proyecto-de-magap-fortalece-la-produccion-de-alevines-de-trucha/

Ministerio de Agricultura, G. A. (27 de octubre de 2015b). www.agricultura.gob.ec. Consultado el 4 de febrero de 2016. Disponible en http://www.agricultura.gob.ec/entregan-10-mil-alevines-de-trucha-a-piscicultores-afectados-por-aluvion-en-oyacachi/ 
Ministerio de Comercio Exterior (2017). Ecuador aumenta sus exportaciones al mundo. Consultado el 11 de marzo de 2016. Disponible en https://www.comercioexterior.gob.ec/ecuador-aumenta-sus-exportaciones-al-mundo/

Mora, V. y Uyaguari, M. (2004). Situación actual de las especies introducidas en el Ecuador con fines acuicolas. Guayaquil: Escuela Superior Politécnica del Litoral.

Muñoz, A. y Civille, G. V. (1998). Universal, product and attribute specific scaling and the development of common lexicons in descriptive analysis. Journal of Sensory Studies, 13, 57-75.

Myhrvold, N. y Young, C. (2010). Modernist cousine. Estados Unidos.

Ommanney, F. (1964). Los peces. México: Offset Multicolor S. A.

Pokniak, J., Cornejo, S., Bravo, I. y Battaglia, J. (2001). Pigmentación de trucha arco iris (oncorhynchus mykiss) tipo mar alimentadas con dos niveles de astaxantina en dietas. Archivos de Medicina Veterinaria, 22(3).

Pozo, R. (1993). Problemas de calidad en la carne de toro lidiado. Real Academia de Córdoba, de Ciencias, Bellas Letras y Nobles Artes, 125,167-182.

Quitral, V. y Pinheiro, A. (2015). Efecto de edulcorantes no calóricos en la calidad sensorial del jugo de naranja. Revista Chilena de Nutrición, 42(1).

Ramírez-Navas, J. S. (2012). Análisis sensorial: pruebas orietadas al consumidor. Recitela, 90-92.

Sabery sabor (abril-mayo de 2014). Consultado el 28 de diciembre de 2016. Disponible en http://www.saberysabor.com/revista-gastronomia/numero/148

Sáez, C. (23 de julio de 2016). El cerebro se sienta a la mesa. La vanguardia.

Sancho, J. (2002). Introducción al análisis sensorial de alimentos.

Van De Vis, H. y Al, E. (2003). Is humane slaughter of fish possible for industry? Aquaculture Research, 34(3), 211-220.

CC BY-NC-ND 